\title{
The response of the magnetosphere-ionosphere system to a sudden dynamic pressure enhancement under southward IMF conditions
}

\author{
Y. Yu and A. J. Ridley \\ Department of Atmospheric, Oceanic and Space Science, University of Michigan, Ann Arbor, MI, USA \\ Received: 24 March 2009 - Revised: 22 October 2009 - Accepted: 26 November 2009 - Published: 2 December 2009
}

\begin{abstract}
The magnetospheric response to step-like solar wind dynamic pressure increases under southward IMF conditions is studied using the University of Michigan MHD code. A two phased response in the ionosphere is observed, similar to what is observed when the IMF is northward by looking into the residual potential and field-aligned current (FAC) patterns in the ionosphere. The first phase response right after the high pressure enhancement hits the magnetopause is associated with a pair of FACs downward in the postnoon and upward in the prenoon region. These FACs are caused by dusk-to-dawn electric fields inside the dayside magnetopause launched by a fast mode compressional wave. The second phase response shows another pair of potential cells as well as FACs in opposite polarity, which originates from magnetospheric vortices on the equatorial plane. The vortices appear to be formed by the recovery of the system from the fast mode wave.
\end{abstract}

Keywords. Ionosphere (Plasma convection) - Magnetospheric physics (Magnetosphere-ionosphere interactions; Solar wind-magnetosphere interactions)

\section{Introduction}

Understanding the interaction between the solar wind and the magnetospheric-ionospheric system is important for a number of practical and theoretical reasons. Space weather basically begins with this interaction. Studies have shown that the interplanetary magnetic field (IMF) strongly controls the state of the magnetosphere (Nishida, 1983). While the solar wind pressure can determine the size of the magnetosphere, less is understood about how the pressure influences the electrodynamics of the magnetosphere-ionosphere system. Many

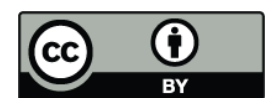

Correspondence to: $\mathrm{Y}$. Yu

(yiqunyu@umich.edu) studies have shown that the solar wind density has little to no effect on the electric potential in the ionosphere, while the velocity can help to control the electric potential (e.g., Gonzalez, 1990; Weimer, 1995; Boyle et al., 1997). Recently, it has been suggested that the solar wind pressure helps to control the energy transfer from the solar wind to the ionosphere (Lopez et al., 2004; Boudouridis et al., 2003) and whether the ionospheric potential will be saturated (Siscoe et al., 2002). While the role of the relatively calm solar wind pressure/density is questionable in driving the magnetosphere, transient or step changes in the pressure or density appear to have a strong influence on the magnetosphere for a short period of time. Magnetic perturbations measured by ground-based magnetometers after pressure changes in the solar wind have been reported by many researchers (FriisChristensen et al., 1988; Farrugia et al., 1989; Sibeck et al., 2003; Araki, 1994; Russell and Ginskey, 1995; Engebretson et al., 1999; Moretto et al., 2002; Sibeck et al., 2003). Ground magnetic perturbations experience two successive impulses that are denoted as PI (preliminary impulse) and MI (main impulse) (Araki, 1994). PI signatures at lower latitudes are positive in the morning sector and negative in the afternoon; MI signatures have the reversed sense. At higher latitudes the magnetic signatures are opposite.

Engebretson et al. (1999) showed equivalent convection patterns calculated from all available magnetometer data for the preliminary impulse and revealed traveling convection vortices (TCV). Moretto et al. (2000) interpreted the response measured by ground-based magnetometers to a compression of the magnetosphere on 22 August 1995 in terms of potential patterns by means of the assimilative mapping of ionospheric electrodynamics (AMIE) technique (Richmond and Kamide, 1988). Slinker et al. (1999) studied the ionospheric response to a density pulse in the solar wind using a global 3-D MHD model. The last two studies showed that a pair of oppositely rotating flow vortices appear in the ionosphere around $70^{\circ}$ near noon and propagate toward the

Published by Copernicus Publications on behalf of the European Geosciences Union. 
nightside, fading as they move. They also reported a second pair of ionospheric convection vortices that follow the first set but rotate in the opposite sense. Slinker et al. (1999) presented results that show a pair of magnetospheric vortices traveling toward the magnetotail that are associated with the ionospheric response.

Past studies have speculated that the ionospheric vortices are driven by field-aligned currents generated at the magnetopause, due to the indentation of the magnetosphere at the pressure front. The perturbations on the magnetopause move along the flanks of the magnetosphere with the solar wind, causing the FACs to travel antisunward, and thus ionospheric traveling vortices result (Glassmeier and Heppner, 1992). Lysak and Lee (1992) studied the response of the magnetosphere to a pressure pulse at the magnetopause using a 3D model of Ultra-Low-Frequency (ULF) waves in a dipole geometry and showed that a compressional wave is directly excited by the pressure pulse, and converts to a shear mode Alfvén wave in the inhomogeneous magnetosphere, carrying the FACs down to the ionosphere. In other words, the FACs are generated inside the magnetosphere but not on the magnetopause. Lühr et al. (1996) proposed that the FACs are generated by local pressure perturbations at the inhomogeneous magnetospheric boundary layer. Slinker et al. (1999) interpreted the ionospheric traveling vortices as a result of fieldaligned currents that are generated by a hydromagnetic wave propagating in the inhomogeneous magnetosphere plasma from the dayside to the nightside. Tamao (1964a), Tamao (1964b) and Southwood and Kivelson (1990) proposed the development of vortical flows in the ionosphere through the conversion of compressional to transverse waves. Kivelson and Southwood (1991) proposed that the field-aligned currents are generated by the shear Alfvén perturbations directly at the boundary. Cowley (2000) reviewed and compared several previously suggested generation mechanisms for traveling convection vortices (TCV) that result from a compressive pulse in the solar wind, and concluded that there is yet no consensus on which of the proposed mechanisms matches the observed pattern for an impulsive compression. Sibeck et al. (2003) suggested that field-aligned currents would be generated where the transient azimuthal pressure gradients are applied to the more permanent radial gradients, and peak at the inner edge of low latitude boundary layer (LLBL).

While these FAC generation mechanisms do not consider separately the two-phased response to the solar wind pressure increase, some studies started looking into the PI and MI phases separately. Engebretson et al. (1999) suggested the first short-lived set of FACs results in charge imbalances, which, when unloading, could help to enhance the effect of the second set of FACs. Araki (1994), Moretto et al. (2000) explained the first response as a result of a dusk-to-dawn inductive electric field launched by a compressional wave that is caused by the sudden change in magnetic field at the dayside magnetopause due to the compression induced by the pressure enhancement. While Araki (1994) attributed the second response to enhanced convection electric fields, Moretto et al. (2000) inferred it as a generic feature of the magnetospheric and ionospheric response to the pressure increase, but did not determine the physical mechanism. Keller et al. (2002) concluded that the first response is consistent with the theory of Kivelson and Southwood (1991) who proposed that Alfvén waves driven by a pressure perturbation near the magnetopause generates the FACs. The authors also suggested that conversion processes between compressional waves and transverse waves directly are driven by magnetopause indentations, and that the shear Alfvén wave is the best candidate of the generator of the FAC in the second phase. Kataoka et al. (2004) studied TCVs caused by a localized density pulse in MHD simulations and concluded that the transient response at the higher latitude is due to the FACs converted from the inertial current associated with the magnetopause deformation via the curvilinear effect, while the lower latitude TCVs result from the FACs that are converted via both curvilinear effect and inhomogeneous effect.

Fujita et al. (2003a), Fujita et al. (2003b) showed simulation results of the response of the geospace environment to a sudden solar wind pressure enhancement under northward IMF conditions. These researchers thoroughly analyzed the generation mechanism by focusing on current systems in the two-phased responses. Fujita et al. (2003a) examined the first response that is associated with the preliminary impulse of ground perturbations by studying the evolution of the ionospheric field-aligned current system and ground-based magnetic perturbations. They showed that an upward (downward) FAC appears in prenoon (postnoon) region, propagating poleward and nightward, and that the northward component of ground-based magnetic field shows bipolar variations. They suggested that the current associated with the preliminary impulse first is generated at the magnetopause and subsequently is converted into field-aligned current toward the ionosphere via polarization current in the compressional wavefront launched by the solar wind impulse. This conversion involves a mode change from the compressional wave to the Alfvén wave and occurs in the region with a steep Alfvén wave speed gradient. Fujita et al. (2003b) showed that during the main phase, another twin convection cell system with opposite rotational sense appears on the dayside at lower latitudes, propagating toward the nightside. The authors studied two successive current systems. One is a transient current system, consisting of three currents: upward FAC on the sunward side, dawn-to-dusk current in the inner magnetosphere that is caused by the deceleration of plasma behind the compressional wavefront, and downward FAC on the night side. While the other one, which is rather stationary and resembles the Region- 1 current system, is driven by a dynamo in the tailward side of cusp region.

While some studies mentioned above did not account for the IMF orientation (e.g., Lysak and Lee, 1992; Engebretson et al., 1999), some studies specifically focused on pressure changes when the IMF was northward (e.g., Russell 
and Ginskey, 1995; Slinker et al., 1999; Moretto et al., 2000; Fujita et al., 2003a,b). Using the University of Michigan MHD code, we have investigated the response of the magnetosphere-ionosphere system to a sudden pressure enhancement under northward IMF conditions and found the same responses as those reported by Slinker et al. (1999), Keller et al. (2002), Fujita et al. (2003a), Fujita et al. (2003b). Here we present results on the magnetospheric response during southward IMF conditions, which is found to be somewhat different than during northward conditions, but having the same two-phased ionospheric response. The generation mechanism of the two phases are discussed in detail.

\section{Methodology}

The magnetohydrodynamic (MHD) code BATS-R-US (Block Adaptive Tree Solar wind Roe-type Upwind Scheme) is described by Powell et al. (1999). It solves the ideal MHD equations and is coupled to an ionospheric electrodynamic model, as described by Ridley et al. (2004). The MHD code has a block-based structure, such that a wide range of scale sizes can be simulated within the same domain. In this work, simulations are conducted with the inner boundary of magnetosphere at $2.5 R_{E}$, resolved with a resolution of $1 / 4 R_{E}$. The initial parameters are IMF $B_{x}=0.5 \mathrm{nT}, B_{y}=0, B_{z}=-5 \mathrm{nT}$, solar wind density $=5 \mathrm{~cm}^{-3}$, temperature $=100000 \mathrm{~K}$, velocity $V_{x}=-400 \mathrm{~km} / \mathrm{s}$, and $V_{y}=V_{z}=0$. The simulation start time is at 16:45 UT. The solar wind conditions are constant until 17:15 UT, at which time a density change from $5 \mathrm{~cm}^{-3}$ to $20 \mathrm{~cm}^{-3}$ is introduced at the upstream boundary of the domain $\left(32 R_{E}\right)$. In order to keep such a contact discontinuity from propagating in the solar wind, the property of continuous thermal pressure across the discontinuity requires the temperature to be $25000 \mathrm{~K}$ after the density impulse, while the other parameters remain unchanged. The simulation runs $30 \mathrm{~min}$ under the disturbed conditions to examine the response of the magnetosphere and ionosphere to the dynamic pressure enhancement.

An estimate of the time delay for the disturbance to propagate before encountering the bow shock is made in this study. Prior to its encounter with the bow shock, the front of the disturbance convects at solar wind speed superposed by the fast mode wave speed that propagates away from the idealized step disturbance. However, the solar wind speed is much larger than the fast-mode wave speed in much of the volume of the magnetosheath and in the solar wind (Farrugia et al., 1989). While numerically the discontinuity diffuses slightly, causing part of the step disturbance to arrive at the bow shock earlier than the real step change, resembling the fast wave perturbation, the diffusion does not introduce a significant difference from a real step change, since the spreading of the discontinuity is observed to be about $0.5 \mathrm{~min}$. Therefore, in what follows, the disturbance is treated to be traveling at the

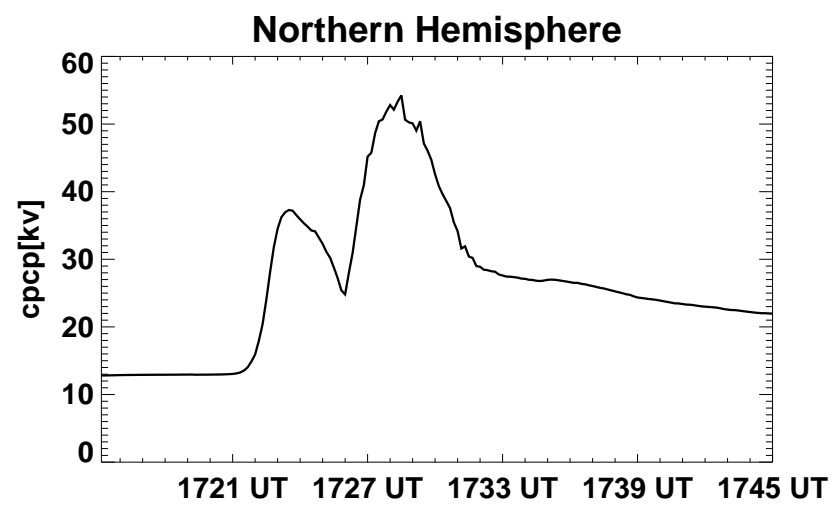

Fig. 1. Cross polar cap potential as a function of time when the high pressure solar wind hits the bow shock around 17:20 UT with northward IMF conditions.

solar wind speed, and the response in the system is caused by the introduced step increase.

\section{Simulation results}

\subsection{Results with northward IMF conditions}

The response of the magnetosphere to a step change in the solar wind density under northward IMF conditions in our simulation results is consistent with previous studies. Two phased responses reported by Fujita et al. (2003a), Fujita et al. (2003b), as described in the Introduction section, are reproduced. Figure 1 shows the cross polar cap potential profile after the sudden pressure enhancement encounters the bow shock around 17:20 UT. The first phase of the response shows an increase of the cross polar cap potential soon after the disturbance hits the magnetosphere, and then it starts to decay 2 min later. This increase of the cross polar cap potential is associated with a strong reversed potential pattern (or enhancement of the northward IMF potential). The second phase of the response takes over in magnitude in another 2 min, significantly increasing before it fades. The potential then begins a phase of slow decline lasting tens of minutes. The potential pattern of the second response is a more typical two-cell pattern, which implies that the currents being driven by the enhanced pressure condition dominate over the northward IMF current system (i.e., the NBZ currents).

\subsection{Results with southward IMF condition}

\subsubsection{Ionospheric signatures}

Under southward IMF conditions, the ionospheric convection is a typical two-cell pattern (positive potential cell on the dawn side, and negative on the dusk side) and the Region1 and Region-2 current systems in the ionosphere have 

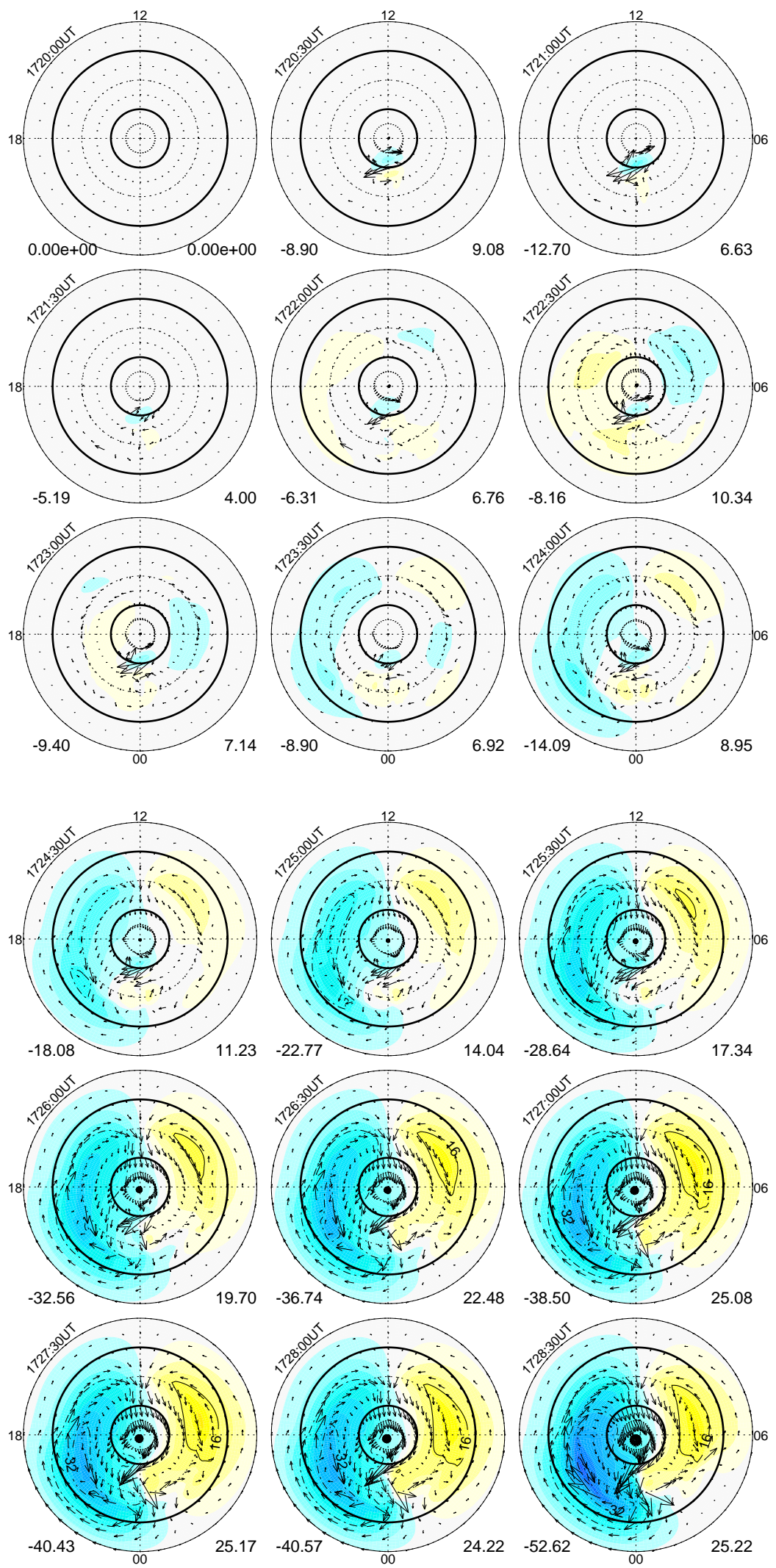

Fig. 2a. Residual potential patterns in the Northern Hemisphere after the pressure enhancement hits the magnetosphere with southward IMF conditions. They are derived by removing a potential pattern, obtained by averaging the patterns from 17:15 UT to 17:20:00 UT, from the plot at the specific time. The yellow (blue) contours represent the positive (negative) residual potential, while the arrows are the equivalent convection velocities. The last plot is the cross-polar cap potential as a function of time. 

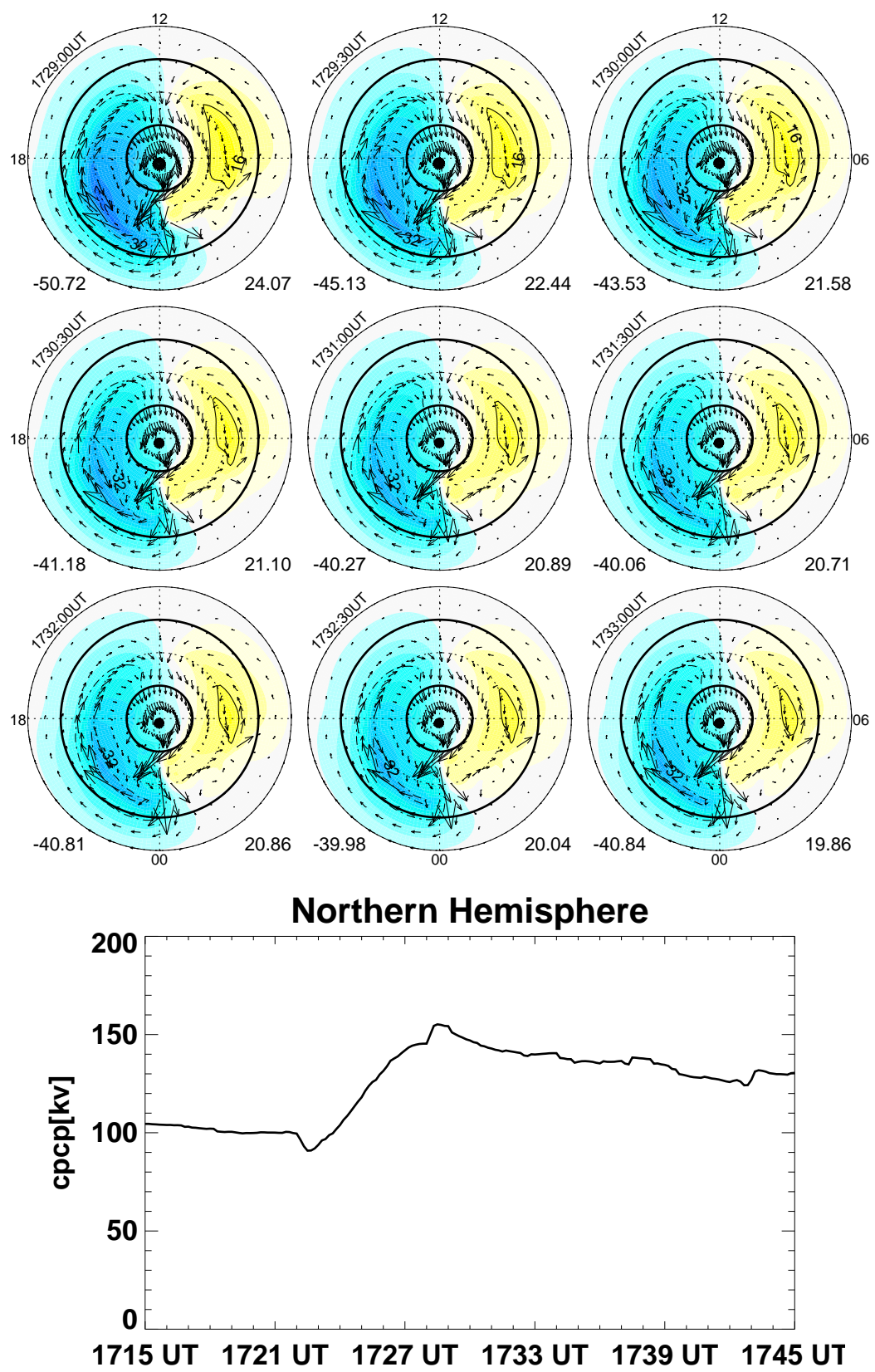

Fig. 2b. Continued.

significant magnitudes. Therefore, in order to detect the response of the ionospheric current and potential to the sudden disturbance, background patterns are subtracted. The backgrounds are obtained by averaging the patterns from 17:15 to 17:20 UT, the time before the sudden pressure enhancement encounters the bow shock, i.e., when the system has not yet been disturbed.

The ionospheric residual potential pattern at 17:20 UT and afterwards at a $30 \mathrm{~s}$ time cadence are shown in Figs. 2a and $2 \mathrm{~b}$. Two weak potential cells, with the positive(negative) cell in the afternoon(morning) sector, appear around 17:22:00 UT at approximately $70^{\circ}$ latitude. The two cells grow and then decay in a time-scale of two minutes, propagating toward the nightside. Another two residual potential cells with the opposite polarity emerge around 17:23:30 UT at $68^{\circ}$ at 10:00 MLT/14:00 MLT. The cells expand and strengthen, propagating slightly toward the nightside. These variations are the same as that shown in Fujita et al. (2003b) and others' work with northward IMF. Although the cross polar cap potential profile (line profile in Fig. 2b) primarily shows a single phased response (an increase from $90 \mathrm{kV}$ up to $160 \mathrm{kV}$ from 17:23 UT to $17: 28 \mathrm{UT}$ ), which corresponds to the second 

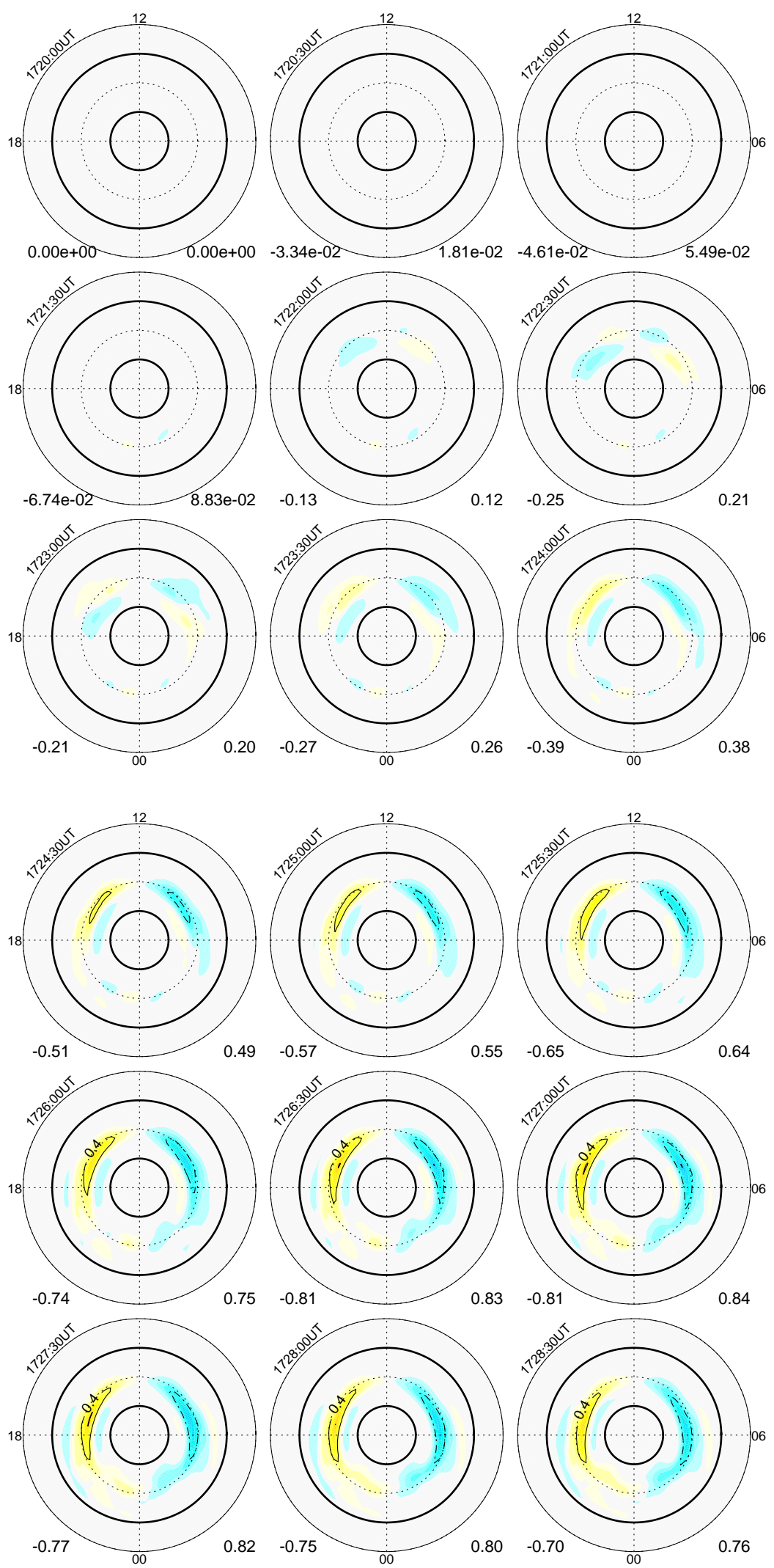

Fig. 3. Residual field-aligned current patterns in the Northern Hemisphere after the pressure enhancement hits the magnetosphere with southward IMF conditions. They are obtained in the same way as that of residual potential patterns. The yellow represents the upward flow of the current, while blue is downward. 

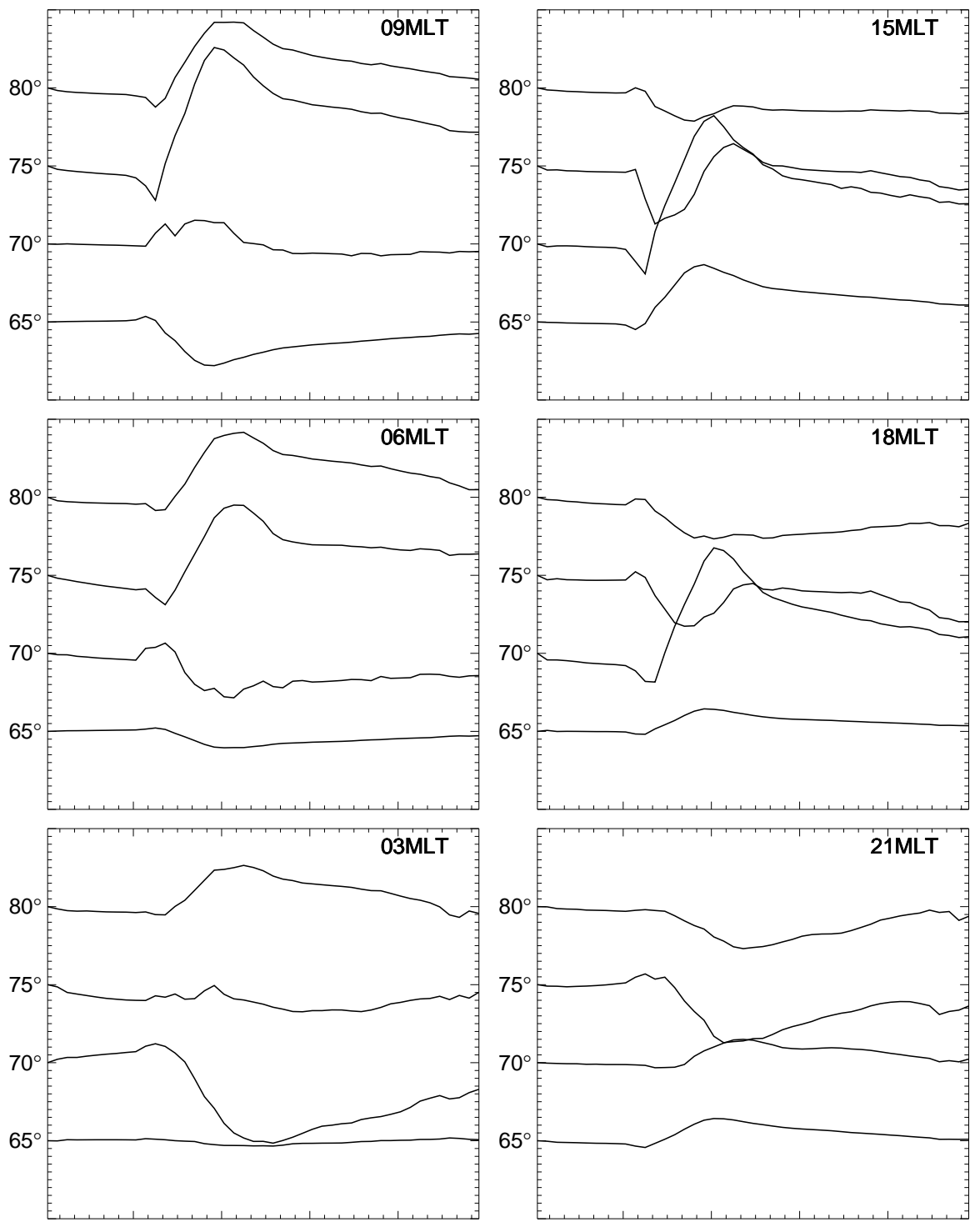

1721 UT 1727 UT 1733 UT 1739 UT

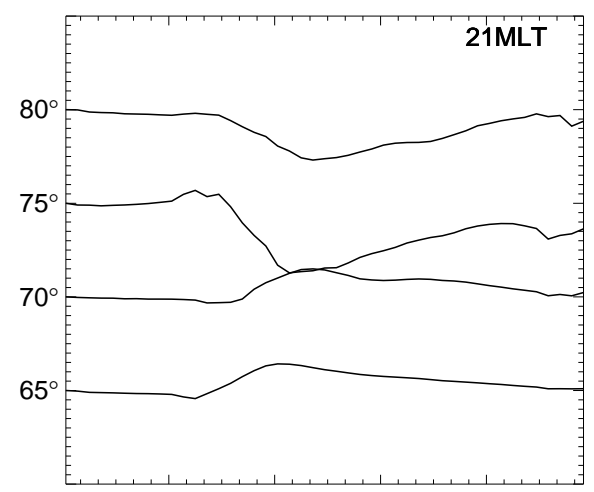

1721 UT 1727 UT 1733 UT 1739 UT

Fig. 4. The $H$ component of the ground magnetic perturbations in the Northern Hemisphere at 6 different longitudes (6 panels) at various latitudes (4 lines in each panel). The interval of perturbations between latitudes is $100 \mathrm{nT}$.

two-cell ionospheric potential set, there is a slight decrease after the magnetopause is disturbed around 17:21 UT with respect to the initial cross polar cap potential. This is the first phase response, as shown in the northward IMF case (Fig. 1). The difference between the northward and southward IMF cases is due to the background ionospheric potential cells. In the northward IMF case, the FACs produced by the magnetospheric response are in the same sense and comparable or larger than the weak background FACs. While in the southward IMF case, the first set of currents is opposite in sense to the background FACs, but significantly weaker than the strong background currents. This causes the net currents (and therefore potential) to be barely changed during the first response. Without subtracting the background pattern, one may not notice a change in the FAC or potential during the first response.

The same two-phased response is also observed in the residual field-aligned current in the ionosphere, as shown in Fig. 3. The residual patterns are obtained in the same way as that for the residual ionospheric potential. The residual downward and upward FAC emerging around 17:22:00 UT in the postnoon and prenoon regions respectively at $73^{\circ}$ are the cause of the residual positive and negative potential cells shown in Figs. $2 a$ and $2 b$. These FACs elongate and propagate at roughly a constant invariant latitude toward the nightside. The second residual current system, resembling the Region- 1 current system, appears near $70^{\circ}$ at 17:22:30 UT on the dayside and propagates in the same 
direction, overwhelming the first FAC set. These results are all consistent with Fujita et al. (2003a), Fujita et al. (2003b) in the sense of ionospheric responses. There is one more residual FAC set that emerges around 17:26 UT at a much lower latitude $\left(65^{\circ}\right)$ : downward FAC elongated in the dusk sector and upward FAC in the dawn sector (too weak to be seen). This residual FAC system is related to the Region2 current, which is observed in Fujita et al. (2005) and our simulation under northward IMF conditions, but the Region2 current in the southward case has a much larger magnitude and appears at an earlier time compared to the northward IMF case.

\subsubsection{Ground signatures}

Ground magnetic field perturbations are calculated from Hall currents over the entire hemisphere by Biot-Savart integral, using the technique described by Yu and Ridley (2008). Figure 4 shows the ground perturbations at various magnetic local times and latitudes. The expected bipolar variation of the preliminary impulse are reproduced at 15:00 MLT and 09:00 MLT around 17:22 UT: positive pulse at higher latitudes in postnoon region and negative at lower latitudes, which is consistent with the first newly emerged ionospheric convection. In addition, the MI signatures are also reproduced, i.e., the deflection goes to negative in the postnoon region at higher latitudes, and the opposite direction at lower latitudes. Fujita et al. (2003b) mentioned that the deflection maximized at different times depending on latitude and MLT. This is reproduced in the simulation reported here with the maximum perturbations from dayside to nightside at various latitudes lagged in time as the wave propagates toward the nightside.

Since FAC is a primary driver of the ionospheric Hall current, which determines the ground-based magnetic perturbations, it is expected that the perturbations will maximize when and where the FACs are strongest. By comparing with Fig. 3, which displays the evolution of FACs in the ionosphere, it is found that the second set of residual FACs maximizes at 08:00 MLT and 16:00 MLT around 17:27:00 UT at $72^{\circ}$ magnetic latitude, which explains the maximum deflection in the ground perturbations at 17:27:00 UT around this latitude at 15:00 MLT and 09:00 MLT. The elongation of FACs towards the nightside contributes to the propagation of the maximum deflection.

\section{Discussion}

It is shown above that the magnetosphere and ionosphere undergo a two-stage response to a step function in pressure during southward IMF. In the ionosphere, the first response is quite rapid and is very weak compared to the background, and therefore may be easily overlooked. Utilizing a background subtraction technique, though, it is easily observed, and is similar to the northward case. The ionospheric convection vortices are strongly related to the field-aligned currents that connect the magnetosphere and the ionosphere.

\subsection{First phase}

As seen from the review in the Introduction section, there are a number of studies that attempt to interpret the generation of FACs after a sudden solar wind pressure increase. However, most of them did not distinguish between the sequences of the two phases. Here, we attempt to investigate the two responses separately to study both generation mechanisms. For the FACs in the first response, one suggestion from earlier work that separately considered two responses is wave mode conversion, which occurs in the nonuniform plasma (e.g., Fujita et al., 2003a). After the sudden compression of the magnetopause, a compressional wave (i.e., a fast-mode wave) is excited at the dayside magnetopause. This compressional wave converts to shear Alfvén wave at the boundary where the plasma has a sharp spatial gradient in the region of $6<\mathrm{L}<7$ in their simulation. The current is then carried down to the ionosphere along the field-lines by Alfvén waves. Another suggestion is from Araki (1994) who proposed a physical model that attributed the first double-cell system to the inductive dusk-to-dawn electric field transmitted to the polar ionosphere from the compressional wavefront propagating in the dayside magnetosphere.

Examining the magnetospheric flow at the beginning of the disturbance helps to understand the first response observed in the ionosphere. It is well known that the dayside reconnection plays a dominant role in influencing the magnetosphere-ionosphere system during steady-state periods under southward IMF conditions (Dungey, 1961). During the first $2 \mathrm{~min}$ after the pressure change encounters the magnetopause, it appears that reconnection plays little role in determine the flow on the dayside magnetosphere. Figure 5 illustrates that the disturbance caused by a sudden pressure increase in the solar wind is opposite to the steady flow that is usually caused by the subsolar reconnection: the convection at the dayside magnetopause cavity is observed to have an Earthward flow just after the pressure enhancement hits the bow shock around 17:20 UT, rather than the typical sunward flow, until 17:23:00 UT when the reconnection site starts to stabilize at a new position and the sunward plasma flow recovers. This sequences is shown in the lower row of Fig. 5, which illustrates the plasma $V_{x}$ in color with the magnetic field-lines overplotted in $\mathrm{Y}=0$ plane. The blue branch inside the dayside magnetosphere cavity (at 17:21:30, 17:22:00 and 17:22:30) represents the antisunward flow soon after the high pressure hits the magnetopause.

The upper row of Fig. 5 shows the electric field in the equatorial plane in the dayside magnetosphere. The color represents the Y-component of the electric field, while the vectors indicate $E_{x y}$. Before the magnetosphere is disturbed, the electric field in the dayside magnetosphere cavity is 


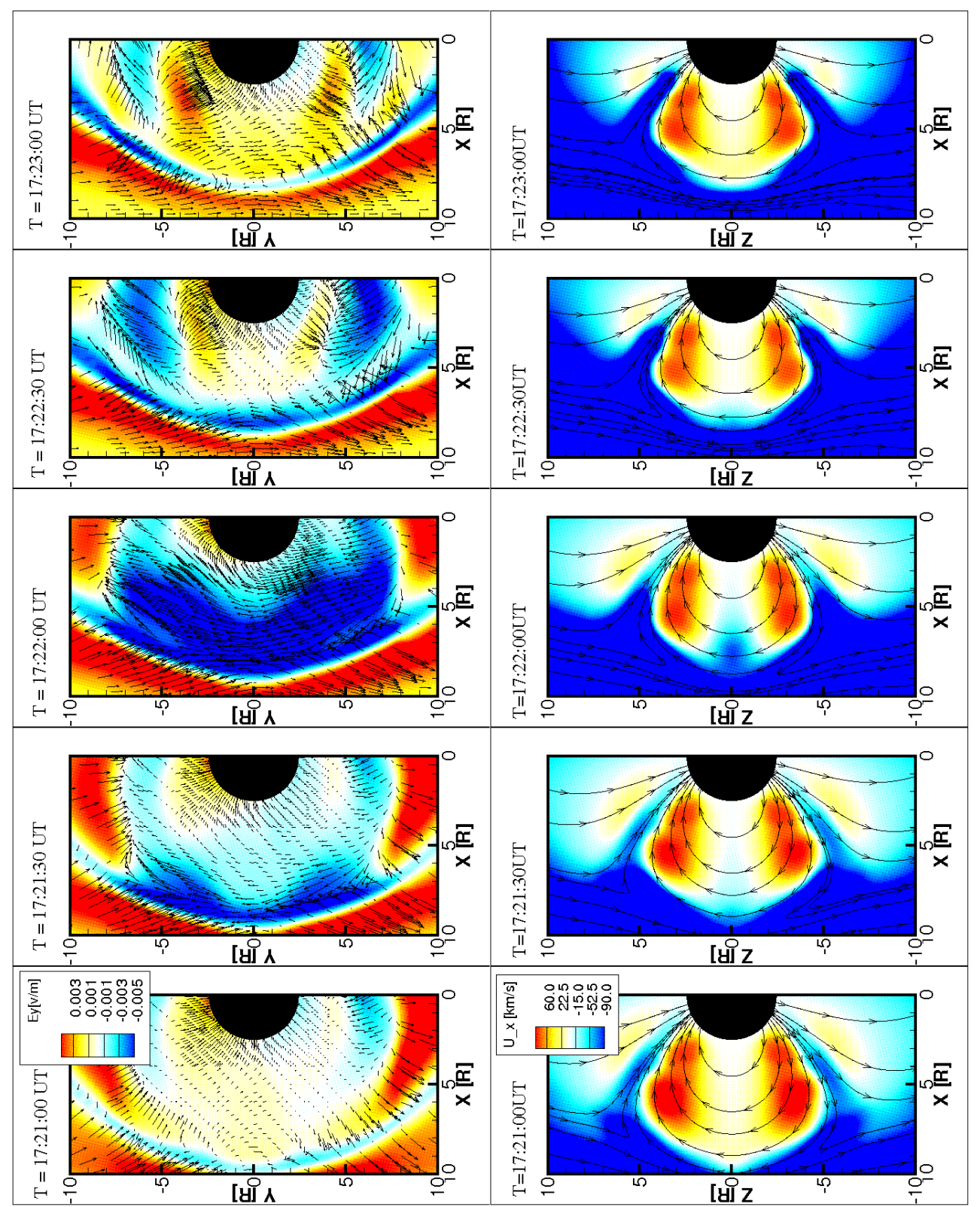

Fig. 5. Upper row: electric fields on equatorial plane. Color represents the dusk-to-dawn (Y) component of eletric fields, and black arrows are vectors of electric fields on the plane (i.e., in both $\mathrm{x}$ and y components). Lower row: Snapshots of $V_{x}$ in the dayside magnetosphere on the $\mathrm{Y}=0$ plane. Color represents the velocity, and the lines are the magnetic field lines. Note that the magnetosphere is slightly stretched in the $\mathrm{x}$ axis compared to the $\mathrm{y}$ axis.

roughly dawn-to-dusk, except in the places where magnetospheric convection vortices reside. After the high pressure enhancement encounters the subsolar magnetopause, duskto-dawn electric fields are created inside the dayside magnetopause, generating antisunward plasma flow, due to the compressional fast mode wave. This fast mode wave is caused by a large $d B / d t$ due to the inward motion of the magnetopause. A dusk-to-dawn displacement current is generated by the change of electric fields.
It should be noted that the reversed convection near the dayside reconnection point seems contradictory to the reconnection process, but the reconnection point is moving Earthward with a velocity of almost $1 / 2 R_{E}$ per minute, (approximately $55 \mathrm{~km} / \mathrm{s}$ ), which is significantly larger than the Earthward flow inside the magnetosphere. Therefore, in the reconnection frame of reference, there is flow directed towards the reconnection site on both sides. 


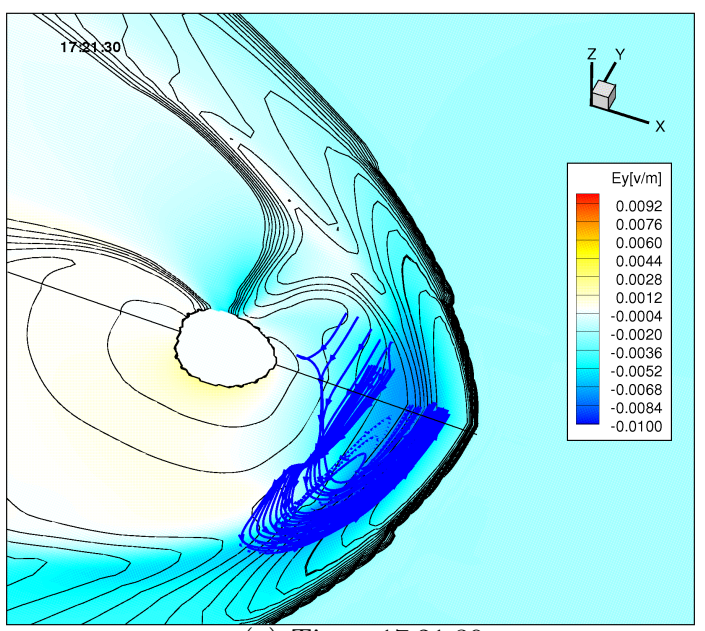

(a) Time: 17:21:30

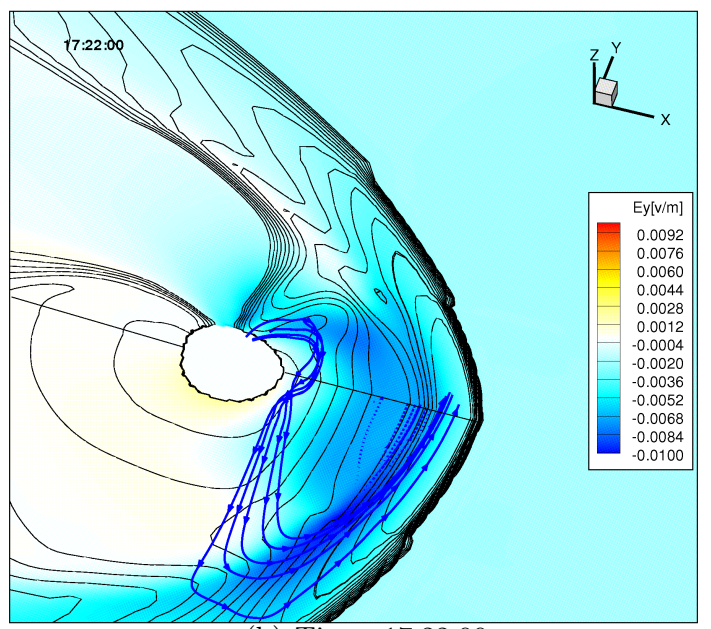

(b) Time: 17:22:00

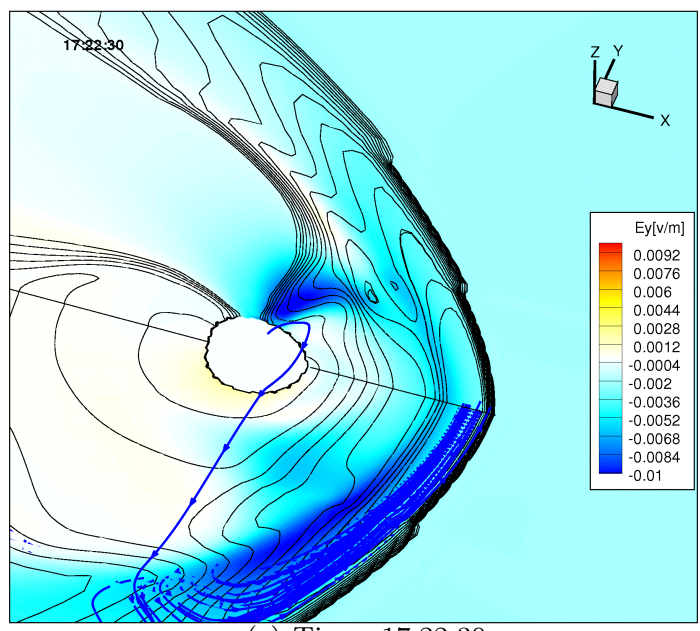

(c) Time: 17:22:30

Fig. 6. The current flow in a 3-D view in the first phase. Blue lines: current flow; Color on the $\mathrm{Z}=0$ and $\mathrm{Y}=0$ plane: electric field in $\mathrm{Y}$ direction; black contour lines: thermal pressure in the magnetosphere. The axis orientation is shown in the upper right corner.
Three-dimensional plots are shown in Fig. 6 to help to investigate the relationship between the first pair of fieldaligned current in the ionosphere and the inductive electric field. After introducing a sudden pressure enhancement in this simulation, the response in the magnetosphereionosphere system is found to be the same as that observed during northward IMF conditions, implying that the first response in the system has little to do with the IMF orientation, as long as the IMF stays steady. Furthermore, it is difficult to distinguish the newly emerged FACs from the background ones due to the same polarity of these FACs under southward IMF conditions. Therefore we choose to show the 3-D results from simulation under northward IMF conditions. The color on $\mathrm{Z}=0$ and $\mathrm{Y}=0$ plane in Fig. 6 is the $\mathrm{Y}$ component of inductive electric field. The black lines are the contour lines of thermal pressure. Blue lines represent the current flow in the magnetosphere. Initially, after the sudden compression, the inductive electric field caused by the fast mode wave generates a dusk-to-dawn displacement current just inside the dayside magnetopause, which is closed by the dawnto-dusk magnetopause current, seen at 17:21:30 UT. During the fast mode wave passage in the dayside magnetosphere, this dusk-to-dawn displacement current is produced in the space between the Earth and dayside magnetopause and follows the propagation of the inductive electric field, which wraps around the Earth from dayside. At 17:22:00 UT, the current circuit is no longer completely in the magnetosphere: the displacement current is now closed by the field-aligned current that flows down towards the ionosphere on the afternoon side (blocked) and out of the ionosphere on the morning side, forming a current loop with the dawn-to-dusk magnetopause current. This field-aligned current pair is consistent with the first response. With the propagation of the inductive electric field towards the nightside, the roots of FACs in the ionosphere also move in that direction. The turning point from displacement current to FAC on the morning side appears around $(5,-3.5,5) R_{E}$, which is at a high $\mathrm{Z}$ position and close to the noon-midnight meridian. Actually, the root of this current flow in the ionosphere lies around $73^{\circ}$ magnetic latitude and 10 magnetic local time, which is consistent with the position where the ionospheric vortex appears.

Some may argue that the turning of the current path from "displacement current-magnetopause current" circuit to "FAC-displacement current-magnetopause current" circuit is a result of the wave mode conversion between the fast mode wave and Alfvén wave at the boundary of great plasma gradient (e.g., $V_{a}$ at the plasmapause in Tamao, 1964a; Fujita et al., 2003a); however, in this simulation, no plasmasphere model is implemented. The structure of the magnetosphere does not display a significant gradient in $V_{a}$ near the position where the displacement current turns to field-aligned current. Furthermore, no time delay is found between this current flow turning to FAC and the ionospheric vortices appearance. They both occur near 17:22:00 UT. While the delay is possible if it is in the wave mode conversion, as the traveling time 

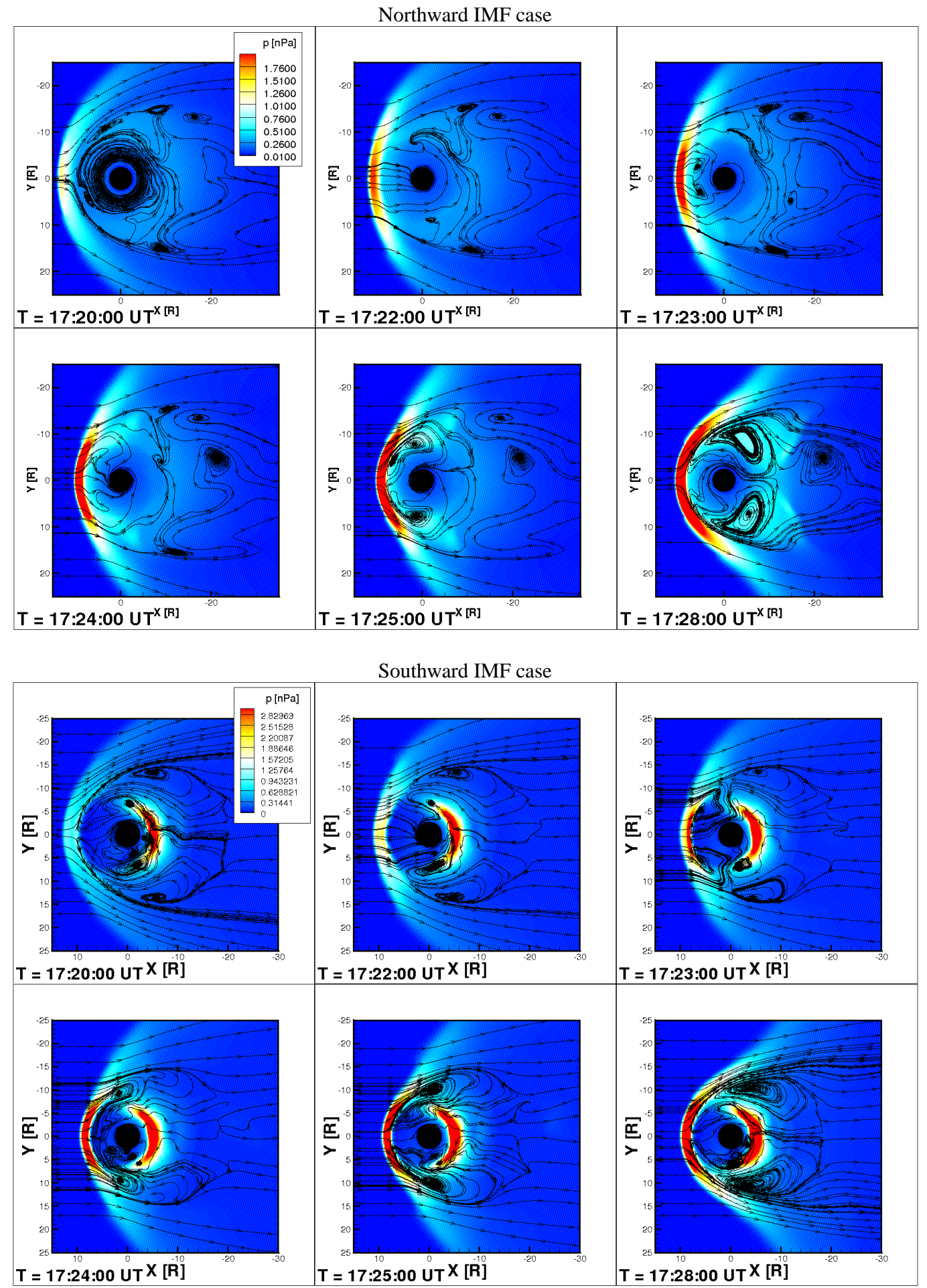

Fig. 7. Magnetospheric convections on the equatorial plane. Upper six panels: the IMF is northward; Lower six panels: the IMF is southward. The color contour represents the thermal pressure.

through Alfvén wave is approximately 1-2 min. Instead, this change of current path might be associated with the enhanced cusp dynamics as a result of fast mode wave propa- gation, since the inductive electric field is observed to travel towards the cusp region where the change of current path occurs. Samsonov et al. (2009) observed a current dynamo 

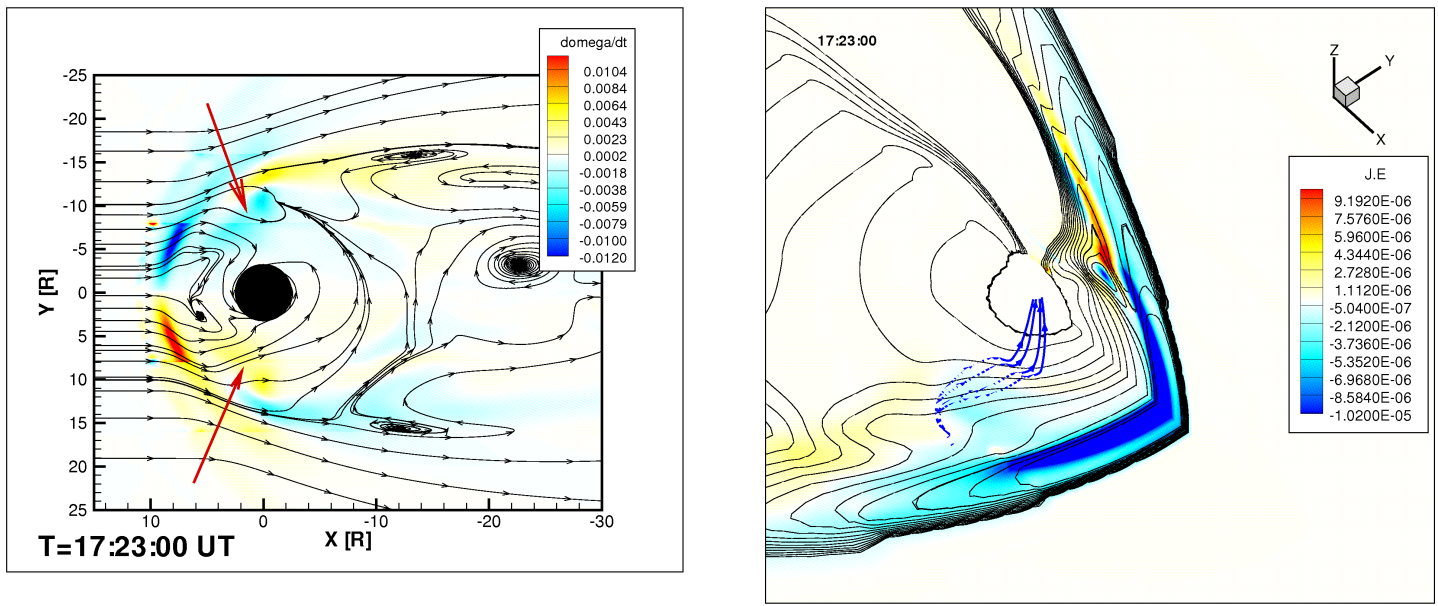

(a) Time 17:23:00
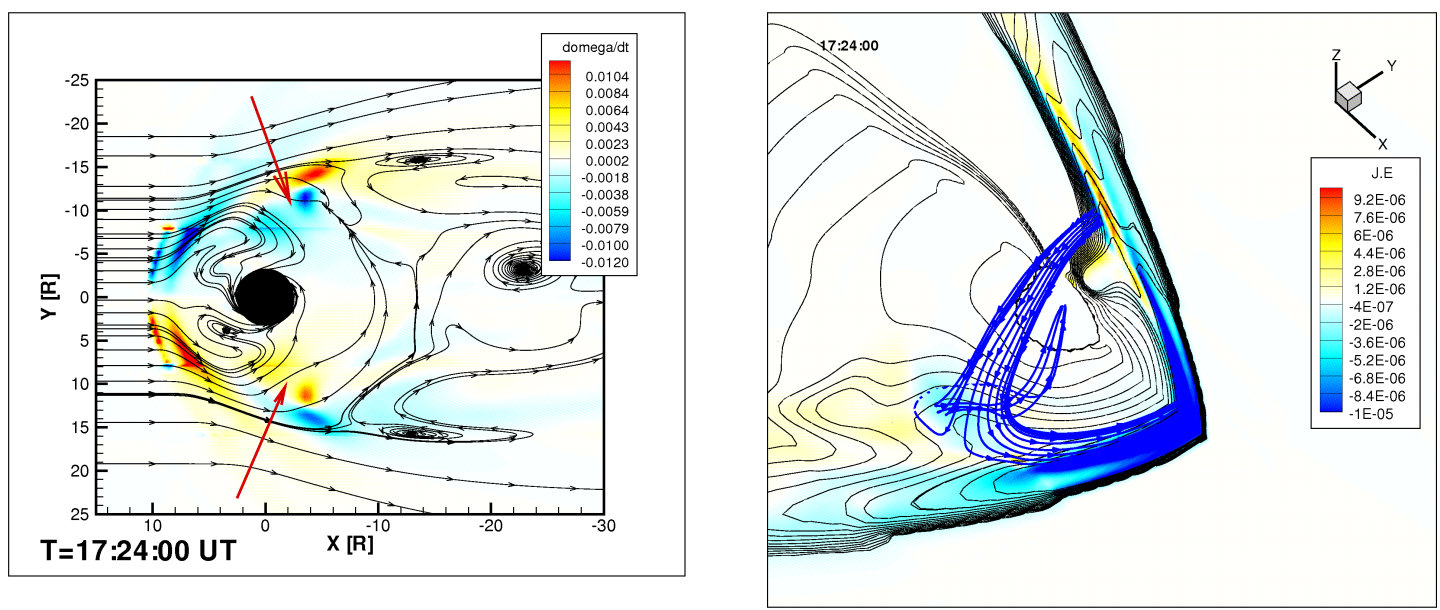

(b) Time 17:24:00
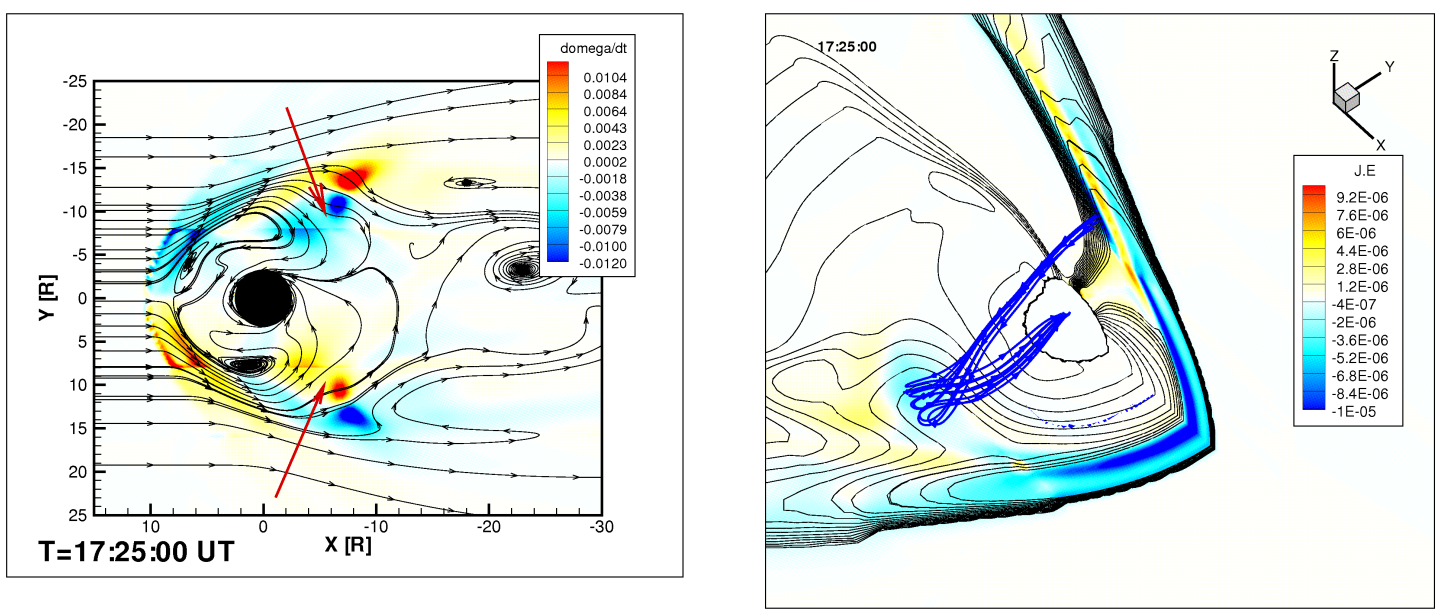

(c) Time 17:25:00

Fig. 8. Northward IMF condition: left column displays the plasma convection (shown by the black streamlines) in the equatorial plane, and the color contour is $\frac{d \Omega_{\|}}{d t}$ (or the 1.h.s. of Eq. 1); right column shows the current flow in 3-D, and the color on $\mathrm{Y}=0$ and $\mathrm{Z}=0$ planes represents $\boldsymbol{J} \cdot \boldsymbol{E}$, while the black contour represents the thermal pressure in the magnetosphere. The unit of $\frac{d \Omega_{\|}}{d t}$ is $10^{-3} / \mathrm{s}$, and unit of $\boldsymbol{J} \cdot \boldsymbol{E}$ is $\mu \mathrm{W} / \mathrm{m}^{3}$. 
near the dayside cusp region after the sudden compression of the magnetosphere in a MHD simulation and found a good temporal correlation with the first ionospheric response.

\subsection{Second phase}

Although the second response in the ionospheric potential and current shows the same phenomena as in the northward IMF conditions simulations, the magnetospheric convection, which may help to reveal the generation mechanisms, is different than during the northward case. To address this difference, Fig. 7 shows the magnetospheric convection development in the equatorial plane for both the northward IMF and southward IMF conditions. The streamline represents the plasma flow, while the color contour displays the thermal pressure.

\subsubsection{Northward IMF}

In the northward IMF situation, after the sudden solar wind pressure change hits the bow shock, the pressure evolution and the vorticity motion inside the magnetosphere show a strong relationship between them. According to Ogino (1986):

$$
\begin{aligned}
& \frac{d \Omega_{\|}}{d t}-\frac{\mu}{\rho} \nabla^{2} \Omega_{\|}-\frac{B^{2}}{\rho} \nabla_{\|} \frac{j_{\|}}{B}=-\frac{2 \mathbf{B} \cdot \nabla p \times \nabla B}{\rho B^{2}}, \\
& \frac{d j_{\|}}{d t}-\frac{\eta}{\mu_{0}} \nabla^{2} j_{\|}-\frac{1}{\mu_{0}} \nabla_{\|} B \Omega_{\|}=0
\end{aligned}
$$

where $B$ is the magnetic field, $\mu$ is the viscosity, $\rho$ is the density, $p$ is the pressure, $\eta$ is the resistivity, $\mu_{0}$ is the vacuum magnetic permeability, $\Omega_{\|}$is the parallel component of vorticity (i.e., along the magnetic field), $j_{\| \mid}$is the parallel component of the current density, and $\nabla_{\|}=(\mathbf{B} \cdot \nabla) / B$. The vorticity is mainly generated by the pressure gradient (i.e., the right hand side of Eq. 1), assuming small viscosity in the magnetosphere and small variation of parallel FAC on the equatorial plane (and thus the 2nd and 3rd terms on the left hand side of Eq. (1) are negligible), and the field-aligned current originates from the vorticity. Details about the inertia term $\frac{d \Omega_{\|}}{d t}$ (i.e., how the vortices, and $J_{\|}$are generated) are discussed below.

When the magnetosonic wave is sweeping through the dayside magnetosphere, two high pressure branches appear just inside the magnetopause on both sides of the Earth just sunward of the dawn-dusk meridian. These high pressure bands appear at around 17:23 UT, as seen in Fig. 7a. The front propagates tailward, generating a pressure gradient pointing sunward, and the gradient of the magnetic field is towards the Earth. These two gradients create a vorticity pointing to the north on the dusk side (out of the plane) and south on the dawn side (into the plane), as shown at 17:24 UT, and thus field-aligned currents flowing into the dawn ionosphere and out of the dusk ionosphere. The pressure gradient propagates tailward around the Earth, driving the vortices in that direction (see 17:28:00 UT).
The left column of Fig. 8 shows the plasma convection (black streamlines) superposed on the color contour of $\frac{d \Omega_{\|}}{d t}$ (or the right hand sides of Eq. 1) for the Northward IMF case. Negative (blue) $\frac{d \Omega_{\|}}{d t}$ means the direction of the vorticity is southward (into the plane), while positive (yellow) represents a northward directed vorticity (out of the plane). The increase of $\left|\frac{d \Omega_{\|}}{d t}\right|$ inside the magnetopause (denoted by red arrows) from the dayside towards the flank and the nightside with time indicates the vortices are under development. As observed in the left column, the vortices (as indicated by the flow lines transferring from antisunward flow along the magnetopause to sunward flow deeper in the magnetosphere) are being led by the increasing $\left|\frac{d \Omega_{\|}}{d t}\right|$ from 17:23:00 UT to 17:25:00 UT. The right column of Fig. 8 shows threedimensional view of current flow with the color contours on $\mathrm{Z}=0$ and $\mathrm{Y}=0$ plane representing $\boldsymbol{J} \cdot \boldsymbol{E}$ and the black contour lines representing the thermal pressure. $\boldsymbol{J} \cdot \boldsymbol{E}$ contours in the equatorial plane indicate a dynamo. The "generator" of current is where $\boldsymbol{J} \cdot \boldsymbol{E}<0$, which means the kinetic energy (or plasma convection) is converted to electromagnetic energy. This occurs in front of the emerging vorticity and is a strong evidence for the vorticity-generated field-aligned current mechanism. As seen from three-dimensional view of current flow, this "dynamo" (negative $\boldsymbol{J} \cdot \boldsymbol{E}$ ) appears from the dayside magnetosphere, generating currents that flow into or out of the ionosphere at auroral latitudes. These currents are closed by Chapman-Ferraro currents. When the dynamo travels from the dayside towards the nightside inside the magnetosphere, the current in the ionosphere also moves towards the nightside, consistent with the phenomena observed in the ionosphere. It is found that the current originating from the vorticity near the equatorial plane does not flow along the field line at first, but in a nearly transverse direction within a thin layer $\left(<1.5 R_{E}\right)$ around the equatorial plane before flowing as field-aligned current. This is diamagnetic current, caused by pressure gradient in magnetized plasma. Therefore, the current generated in the vorticity connects with the FAC toward the Earth via this transverse diamagnetic current around the Equatorial plane.

The second phase is in the same sense as the Region-1 current system, and is therefore often considered as an enhancement to the convection pattern (e.g., Boudouridis et al., 2008). It should be noted that this is exactly the same generation mechanism that creates the Region- 2 current system under southward IMF circumstances, except that in the region 2 current system, the high pressure is on the nightside (centered around midnight) typically, instead of centered near the dayside and spreading towards midnight in this case (i.e., the $\nabla P$ term in Eq. (1) has the opposite sign). It should further be noted that this result implies that during northward IMF, a pressure pulse can directly inject cold dense plasma into the plasma sheet over just a few minutes. 

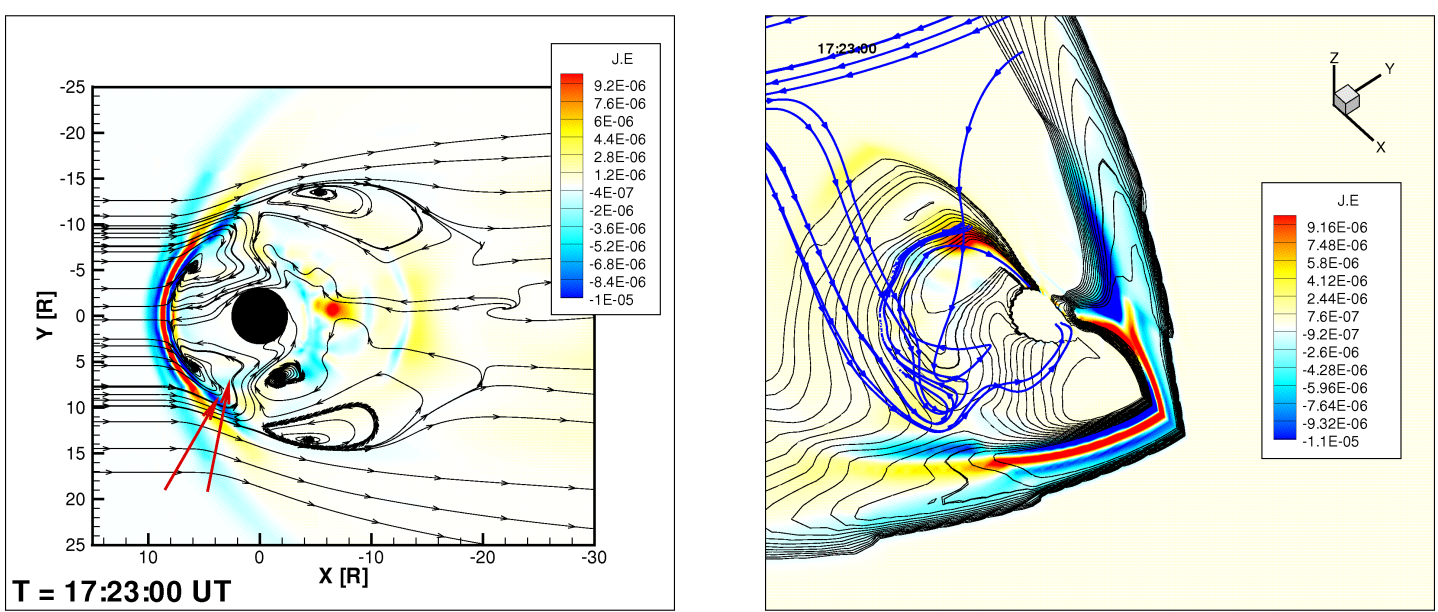

(a) Time 17:23:00
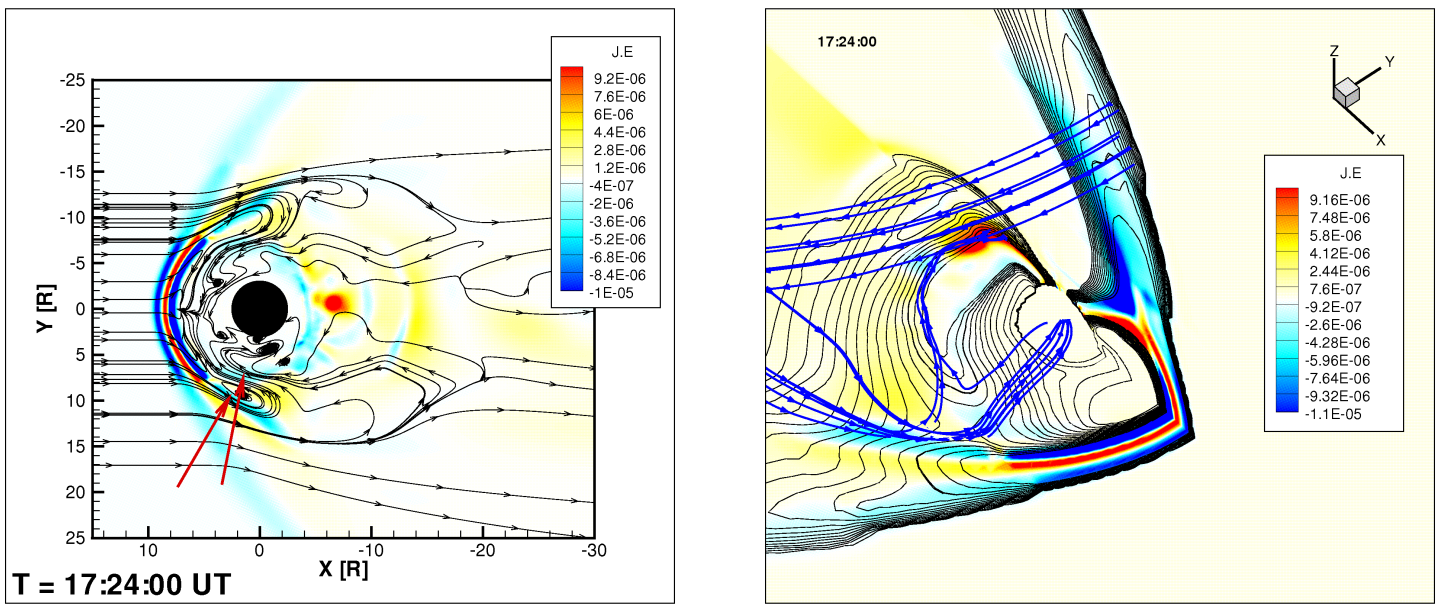

(b) Time 17:24:00
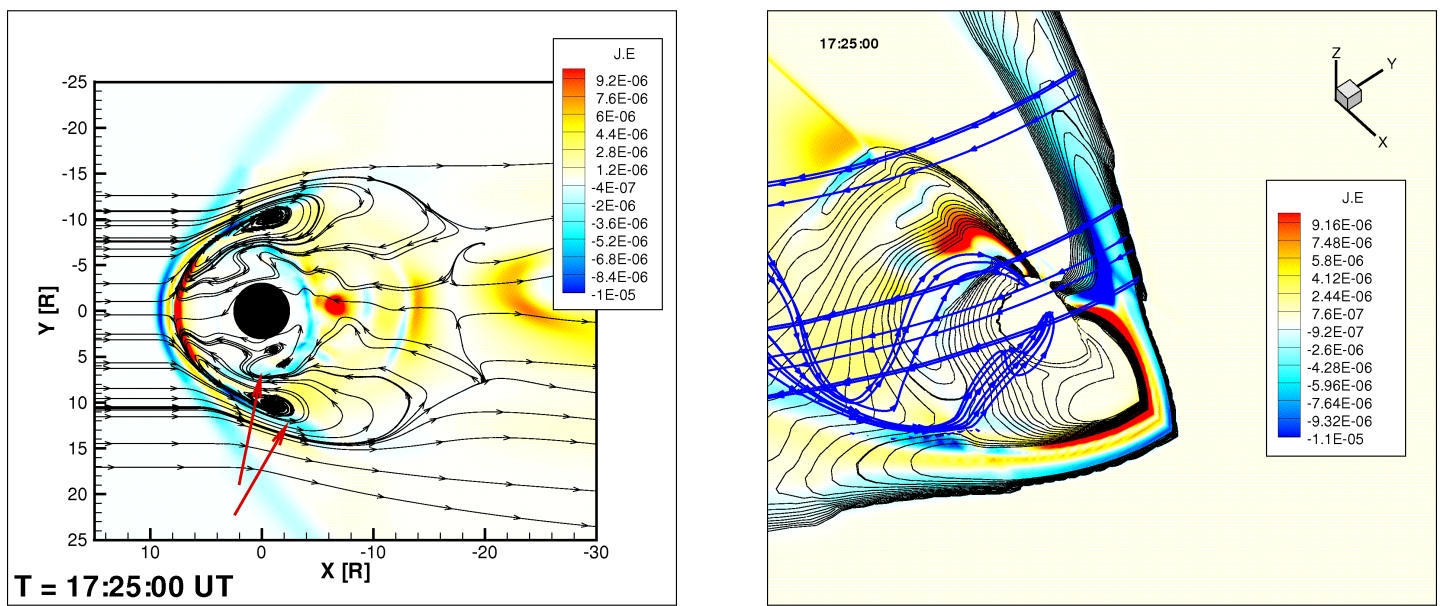

(c) Time 17:25:00

Fig. 9. Southward IMF condition: left column shows the plasma convection (streamlines) in the equatorial plane, with the contour representing $\boldsymbol{J} \cdot \boldsymbol{E}$; right column shows the 3-D current flow in the same format as in the right column of Fig. 8. 


\subsubsection{Southward IMF}

Under southward IMF conditions (Fig. 7b), however, the pressure pattern of the magnetosphere develops differently. No high pressure branches appear inside the magnetosphere. Instead, the high solar wind pressure merely compresses the magnetosphere, and enhances the bow shock and nightside ring current. The absence of high pressure branches is because the enhanced reconnection keeps the plasma from entering the dayside magnetosphere, and the ring current increases on the nightside, preventing the high pressure from wrapping around the Earth towards the nightside. Therefore, vortices behave in a different manner: no pressure gradient driven vortices are observed to propagate around the Earth and towards the nightside inside the magnetosphere. Instead, the dayside portion of the existing vortices inside the magnetospheric flank are destroyed by the inflow plasma (compare 17:20:00 and 17:22:00 at the dayside magnetopause), followed by rebuilding of this part by the shear flow along the magnetopause (see 17:23:00 UT and beyond). The flow lines switch to almost completely oriented in the Y direction near the magnetopause at dawn and dusk at 17:23:00. While these flow patterns are not completely closed, there is a strong indication that this is the start of the vorticity that results in closed cells starting at 17:24 and 17:25 UT.

The left column of Fig. 9 shows the plasma convection with $\boldsymbol{J} \cdot \boldsymbol{E}$ as color contours on the equatorial plane in the Southward IMF case. It is found that two pairs of dynamo currents (in blue) emerge from the dayside magnetosphere (denoted by the red arrows): One inside the magnetopause flank and the other one in the inner magnetosphere. They correspond to the rebuilding of the large-scale vortex along the magnetopause flank and the rebuilding of the smallerscale vortex in the inner magnetosphere, which are associated with the new field-aligned current in the second phase and the Region-2 current system respectively. 3-D current flow is shown in the right column of Fig. 9, with $\boldsymbol{J} \cdot \boldsymbol{E}$ on $\mathrm{Y}=0$ and $\mathrm{Z}=0$ plane. The current generated from the dynamo on the magnetopause flank flows into the ionosphere on the morning side and out of the ionosphere on the afternoon side. This FAC is closed by the magnetopause current. The diamagnetic current again is observed around the Equatorial plane near the pressure gradient on the flank, connecting the magnetopause current and the field-aligned current. The tailward motion of this dynamo drives the same motion of the second pair of FACs in the ionosphere.

In the inner magnetosphere, the initial convection, which is related to the Region-2 current system, is partially destroyed by the antisunward plasma flow during the period when the fast mode wave sweeps through the dayside magnetosphere (i.e., the inner magnetosphere is under-shielded from approximately 17:21 UT-17:25 UT). After that, the two convection vortices in the inner magnetosphere start to recover and strengthen starting at 17:24 UT (see Fig. 7b). Since the residual Region-2 currents appear in the ionosphere around 17:26 UT (see Fig. 3), the time difference indicates that it takes about two minutes or so for the Region-2 current to be restored and then exceed the original magnitude. These convection vortices are created by the pressure gradient that is generated by dense plasmasheet on the nightside. Both the Region- 1 and 2 currents are enhanced in magnitude under the new configuration.

\subsubsection{Cross polar cap potential features}

Note that the cross polar cap potential increases up to $160 \mathrm{kV}$ at 17:28 UT by the sudden compression before leveling off when the high-pressure solar wind sweeps through the magnetosphere. This transient increase can be explained by the elastic characteristic of the plasma (Russell and Ginskey, 1995); the magnetosphere in this simulation is observed to be over-compressed at first and then to relax to a fixed size. Correspondingly, the vortices experience enhancement and decrease subsequently, which then influences the field-aligned current that connects the ionosphere and the magnetosphere and thus the ionospheric electric potential. The time when the magnetosphere is most compressed is found to be well correlated with the time when the FACs in the ionosphere maximize. Between these two times, a communication time of 1-2 min is allowed as the FACs is carried by Alfvén wave from the equatorial magnetosphere down towards the ionosphere. The transient increase in the ionospheric cross polar cap potential during southward IMF is not accounted for in simple empirical relationships between the solar wind/IMF and the ionospheric potential (e.g., Boyle et al., 1997). Further, it has been noted by some that the potential should decrease if the pressure is increased, due to the decrease in the length of the reconnection line (e.g., Ridley, 2005). These simulation results show that under transient conditions, the potential may increase significantly. This effect was discussed by Boudouridis et al. (2004), when comparing to the Siscoe et al. (2002) formulation. It is clear that during the transient time, these simple relationships do not represent the true physics that is occurring in the magnetosphere, and a more sophisticated technique, such as that described here, needs to be utilized. Conversely, a time-dependent empirical relationship could be developed that specifically includes the effects of pressure increases and decreases. Further, it is uncertain how long the magnetosphere is disturbed because of this transient behavior, and whether the magnetosphere actually settles down to its initial state or a different state after a long enough time period. These are issues that may be addressed in further studies.

Besides the transient feature of the cross polar cap potential, another feature is that the cross polar cap potential is around $130 \mathrm{kV}$ in the new configuration, significantly higher than the initial value (i.e., $\sim 100 \mathrm{kV}$ ), although this potential gradually decreases near the end of the simulation. Sonnerup and Siebert (2003) pointed out that the FACs at the dusk and dawn sides are larger since the shear flow inside 
the magnetospheric flank is larger, which is consistent with the simulation results. As the forces such as $\boldsymbol{J} \times \boldsymbol{B}$ and $\nabla p$, which control the plasma shear flow on the magnetospheric flank, are enhanced due to several reasons (i.e., the increased magnetic field in the compressed magnetosphere, the increased magnetopause currents and the larger pressure gradient), the shear flow along the magnetopause is enhanced in the new configuration at the end of the simulation, resulting in enhanced FACs, and thus a higher cross polar cap potential in the recovery phase.

On the other hand, the gradual decrease of the cross polar cap potential near the end of the simulation is possibly caused by energy dissipation between the magnetosphere and the ionosphere. Another possibility is that the ongoing compression in the tail magnetosphere depletes part of energy out from the tail region, resulting in the decrease of the cross polar cap potential.

\section{Conclusions}

In this paper, we have simulated the response of the magnetosphere-ionosphere system to a sudden pressure enhancement under southward IMF conditions using the University of Michigan MHD code. The response is found to be similar to that when IMF is northward and consistent with previous studies (e.g. Fujita et al., 2003a,b). In other words, the magnetosphere and ionosphere show a two-phase response under southward IMF conditions when a solar wind density increase encounters the magnetopause. This can be observed by examining the residual patterns of the potential and field-aligned currents in the ionosphere. However, with a strong background potential during the southward IMF, the cross polar cap potential only displays a small decrease during the first phase, and then a large increase in the second phase.

The first response has a negative potential cell in the prenoon region and positive one in the post-noon region around $\sim 70^{\circ}$ soon after the high pressure enhancement encounters the magnetosphere. They travel rapidly toward the nightside and decay. This response is caused by field-aligned currents that are connected with the dusk-to-dawn displacement current caused by the fast mode compressional wave propagating antisunward through the dayside magnetosphere. The second response appears about 2 min later: another pair of potential cells of the opposite polarity emerge around the same place, but at a slightly lower latitude. These two cells strengthen significantly, supplanting the previous cells, and subsequently decay. This response is associated with the magnetospheric convection vortices, which, under northward IMF conditions, are generated by pressure gradients inside the magnetosphere (i.e., the same mechanism that causes the region 2 currents on the nightside, but with a pressure bulge on the dayside instead). While during southward IMF conditions, this response is caused by the recovery of the equatorial magnetospheric vortices from the inductive electric field (due to $d B / d t$ ) generated by the first response. Because the convection reverses during the first response, the previous pattern needs to be reestablished, which creates vortices close to the magnetopause (one on each side of noon). These vortices spread antisunward, reestablishing the previously existing magnetospheric convection pattern. Field-aligned current, responsible for the second phase in both southward and northward cases, is generated in the vortices and is closed by the magnetopause current via a transverse diamagnetic current around the Equatorial plane.

Acknowledgements. The authors thank Mike Liemohn for his assistance in interpreting the simulation results. This work is supported by NSF grant 0639336 .

Topical Editor I. A. Daglis thanks A. Boudouridis and S. Fujita for their help in evaluating this paper.

\section{References}

Araki, T.: A Physical Model of the Geomagnetic Sudden Commencement, in: Solar Wind Sources of Magnetospheric UltraLow-Frequency Waves, edited by: Engebretson, M. J., Takahashi, K., and Scholer, M., p. 183, 1994.

Boudouridis, A., Zesta, E., Lyons, R., Anderson, P.-C., and Lummerzheim, D.: Effect of solar wind pressure pulses on the size and strength of the auroral oval, J. Geophys. Res. (Space Physics), 108, 8012, doi:10.1029/2002JA009373, 2003.

Boudouridis, A., Zesta, E., Lyons, L., and Anderson, P.: Evaluation of the Hill-Siscoe transpolar potential saturation model during a solar wind dynamic pressure pulse, Geophys. Res. Lett., 31, L23802, doi:10.1029/2004GL021252, 2004.

Boudouridis, A., Zesta, E., Lyons, L. R., Anderson, P. C., and Ridley, A. J.: Temporal evolution of the transpolar potential after a sharp enhancement in solar wind dynamic pressure, Geophys. Res. Lett., 35, 2101, doi:10.1029/2007GL031766, 2008.

Boyle, C., Reiff, P., and Hairston, M.: Empirical polar cap potentials, J. Geophys. Res., 102, 111-126, 1997.

Cowley, S.: Magnetosphere-ionosphere interactions: A tutorial review, Geophys. Monogr. Ser., 118, 91-106, 2000.

Dungey, J.: Interplanetary Magnetic Field and the Auroral Zones, Phys. Rev. Lett., 6, 47-48, 1961.

Engebretson, M. J., Murr, D. L., Hughes, W. J., Lühr, H., Moretto, T., Posch, J. L., Weatherwax, A. T., Rosenberg, T. J., Maclennan, C. G., Lanzerotti, L. J., Marcucci, F., Dennis, S., Burns, G., Bitterly, J., and Bitterly, M.: A multipoint determination of the propagation velocity of a sudden commencement across the polar ionosphere, J. Geophys. Res., 104, 22433-22452, doi: 10.1029/1999JA900237, 1999.

Farrugia, C. J., Freeman, M. P., Cowley, S. W. H., Southwood, D. J., Lockwood, M., and Etemadi, A.: Pressure-driven magnetopause motions and attendant response on the ground, Planet. Spacr Sci. 37, 589-607, doi:10.1016/0032-0633(89)90099-8, 1989.

Friis-Christensen, E., McHenry, M. A., Clauer, C. R., and Vennerstrom, S.: Ionospheric travelling convection vorticies observed near the polar cleft: A triggered response to sudden changes in the solar wind, Geophys. Res. Lett., 15, 253-256, 1988. 
Fujita, S., Tanaka, T., Kikuchi, T., Fujimoto, K., Hosokawa, K., and Itonaga, M.: A numerical simulation of the geomagnetic sudden commencement: 1. Generation of the field-aligned current associated with the preliminary impulse, J. Geophys. Res. (Space Physics), 108, 1416, doi:10.1029/2002JA009407, 2003a.

Fujita, S., Tanaka, T., Kikuchi, T., Fujimoto, K., and Itonaga, M.: A numerical simulation of the geomagnetic sudden commencement: 2. Plasma processes in the main impulse, J. Geophys. Res. (Space Physics), 108, 1417, doi:10.1029/2002JA009763, 2003b.

Fujita, S., Tanaka, T., and Motoba, T.: A numerical simulation of the geomagnetic sudden commencement: 3. A sudden commencement in the magnetosphere-ionosphere compound system, J. Geophys. Res. (Space Physics), 110, 11203, doi:10.1029/ 2005JA011055, 2005.

Glassmeier, K. H. and Heppner, C.: Traveling magnetospheric convection twin vortices: Another case study, global characteristics, and a model, J. Geophys. Res., 97, 3977-3992, 1992.

Gonzalez, W.-D.: A unified view of solar wind-magnetosphere coupling function, Planet. Space Sci., 38, 627-632, 1990.

Kataoka, R., Fukunishi, H., Fujita, S., Tanaka, T., and Itonaga, M.: Transient response of the Earth's magnetosphere to a localized density pulse in the solar wind: Simulation of traveling convection vortices, J. Geophys. Res. (Space Physics), 109, 3204, doi:10.1029/2003JA010287, 2004.

Keller, K. A., Hesse, M., Kuznetsova, M., Rastätter, L., Moretto, T., Gombosi, T. I., and DeZeeuw, D. L.: Global MHD modeling of the impact of a solar wind pressure change, J. Geophys. Res. (Space Physics), 107, 21-1, doi:10.1029/2001JA000060, 2002.

Kivelson, M. G. and Southwood, D. J.: Ionospheric traveling vortex generation by solar wind buffeting of the magnetosphere, J. Geophys. Res., 96, 1661-1667, 1991.

Lopez, R. E., Wiltberger, M., Hernandez, S., and Lyon, J. G.: Solar wind density control of energy transfer to the magnetopause, Geophys. Res. Lett., 8804, doi:10.1029/2003GL018780, 2004.

Lühr, H., Lockwood, M., Sandholt, P. E., Hansen, T. L., and Moretto, T.: Multi-instrument ground-based observations of a travelling convection vortices event, Ann. Geophys., 14, 162181,1996 , http://www.ann-geophys.net/14/162/1996/.

Lysak, R. L. and Lee, D.-H.: Response of the dipole magnetosphere to pressure pulses, Geophys. Res. Lett., 19, 937-940, 1992.

Moretto, T., Ridley, A. J., Engebretson, M. J., and Rasmussen, O.: High-latitude ionospheric response to a sudden impulse event during northward IMF conditions, J. Geophys. Res., 105, 25212532, doi:10.1029/1999JA900475, 2000.

Moretto, T., Hesse, M., Yahnin, A., Ieda, A., Murr, D., and Watermann, J. F.: Magnetospheric signature of an ionospheric traveling convection vortex event, J. Geophys. Res. (Space Physics), 107, 5-1, doi:10.1029/2001JA000049, 2002.

Nishida, A.: IMF control of the Earth's magnetosphere, Space Sci. Rev., 34, 185-200, 1983.

Ogino, T.: A three-dimensional MHD simulation of the interaction of the solar wind with the earth's magnetosphere - The generation of field-aligned currents, J. Geophys. Res., 91, 6791-6806, 1986.
Powell, K. G., Roe, P. L., Linde, T. J., Gombosi, T. I., and Zeeuw, D. L. D.: A solution-adaptive upwind scheme for ideal magnetohydrodynamics, J. Comp. Phys., 154, 284-309, 1999.

Richmond, A. D. and Kamide, Y.: Mapping Electrodynamic features of the high-latitude ionosphere from localized observations: Technique, J. Geophys. Res., 93, 5741-5759, 1988.

Ridley, A. J.: A new formulation for the ionospheric cross polar cap potential including saturation effects, Ann. Geophys., 23, 35333547, 2005, http://www.ann-geophys.net/23/3533/2005/.

Ridley, A. J., Gombosi, T. I., and DeZeeuw, D. L.: Ionospheric control of the magnetosphere: conductance, Ann. Geophys., 22, 567-584, 2004, http://www.ann-geophys.net/22/567/2004/.

Russell, C. T. and Ginskey, M.: Sudden impulses at subauroral latitudes: Response for northward interplanetary magnetic field, J. Geophys. Res., 100, 23695-23702, 1995.

Samsonov, A., Sibeck, D., and Yu, Y.: Transient changes in magnetospheric-ionospheric currents caused by interplanetary shock passage: 1. Northward IMF case, J. Geophys. Res. (Space Physics), in review, 2009.

Sibeck, D. G., Trivedi, N. B., Zesta, E., Decker, R. B., Singer, H. J., Szabo, A., Tachihara, H., and Watermann, J.: Pressure-pulse interaction with the magnetosphere and ionosphere, J. Geophys. Res. (Space Physics), 108, 1095, doi:10.1029/2002JA009675, 2003.

Siscoe, G. L., Erickson, G., Sonnerup, B., Maynard, N., Schoendorf, J., Siebert, K., Weimer, D., White, W., and Wilson, G.: Hill model of transpolar potential saturation: Comparisons with MHD simulations, J. Geophys. Res., 107, 1321, doi:10.1029/2001JA009176, 2002.

Slinker, S. P., Fedder, J. A., Hughes, W. J., and Lyon, J. G.: Response of the ionosphere to a density pulse in the solar wind: simulation of traveling convection vortices, Geophys. Res. Lett., 26, 3549-3552, doi:10.1029/1999GL010688, 1999.

Sonnerup, B. and Siebert, K.: Theory of the low-latitude boundary layer and its coupling to the ionosphere: A tutorial review, Geophys. Monogr., 133, 13-32, 2003.

Southwood, D. J. and Kivelson, M. G.: The magnetohydrodynamic response of the magnetospheric cavity to changes in solar wind pressure, J. Geophys. Res., 95, 2301-2309, 1990.

Tamao, T.: Hydromagnetic interpretation of geomagnetic SSC, Rep. Ionos. Space Res. Janpan, 18, 16-31, 1964a.

Tamao, T.: The structure of three-dimensional hydromagnetic waves in a uniform cold plasma, J. Geomag. Geoelect., 18, 89114, 1964b.

Weimer, D. R.: Models of high-latitude electric potentials derived with a least error fit of spherical harmonic coefficients, J. Geophys. Res., 100, 19595-19608, 1995.

Yu, Y. and Ridley, A. J.: Validation of the space weather modeling framework using ground-based magnetometers, Space Weather, 6, 5002, doi:10.1029/2007SW000345, 2008. 